Stephanie Wolff

Sabine Klatt

Jens C. Wolff

Jochen Wilhelm

Ludger Fink

Manfred Kaps

Bernhard Rosengarten

\section{Endotoxin-induced gene expression differences in the brain and effects of iNOS inhibition and norepinephrine}

Received: 6 June 2008

Accepted: 10 November 2008

Published online: 21 January 2009

(C) The Author(s) 2009. This article is published with open access at

Springerlink.com

This article is discussed in the editorial available at: doi:10.1007/s00134-009-1395-6.

S. Wolff $(\square) \cdot$ S. Klatt · M. Kaps ·

B. Rosengarten

Department of Neurology,

Justus-Liebig-University Giessen,

Am Steg 14, 35392 Giessen, Germany

e-mail: Stephanie.Wolff@neuro.med.unigiessen.de

Tel.: +49-641-9945300

Fax: +49-641-9945309

\section{J. C. Wolff}

Max-Planck-Institute for Heart and Lung Research, 61231 Bad Nauheim, Germany
J. Wilhelm · L. Fink

Department of Pathology, Justus-Liebig-

University Giessen, 35392 Giessen,

Germany

Abstract Purpose: We studied gene expression differences in brain homogenate, hippocampus, somatosensory cortex and cerebellum of rats suffering from sepsis-associated delirium and analyzed the effects of norepinephrine and 1,400 W (specific inhibitor of the inducible nitric-oxide synthase). Methods: We applied microarray screenings to rat brain homogenate 1,3 and $4.5 \mathrm{~h}$ after lipopolysaccharide (LPS, $5 \mathrm{mg} / \mathrm{kg}$ ) or $0.9 \% \mathrm{NaCl}$ treatment. Therapy groups were analyzed after $4.5 \mathrm{~h}$. Validations and compartment specific investigations were carried out by real-time PCR. Results: Most striking gene expression differences were seen $4.5 \mathrm{~h}$ after LPS administration, especially within the hippocampus (chemokines and endothelial cellspecific molecule 1). Norepinephrine resulted in a discrete chemokine upregulation, while $1,400 \mathrm{~W}$ had hardly any effect. Conclusion: Strongest gene regulations were found within the hippocampus. Norepinephrine showed a tendency of having a proinflammatory influence, while $1,400 \mathrm{~W}$ had no clear-cut effect onto the gene expression level.

Keywords Sepsis - Delirium · Encephalopathy · Hippocampus . Chemokines · Microarrays

\section{Introduction}

Sepsis-associated delirium (Diagnostic and statistical Manual, DSM IV; International Classification of Diseases, ICD 10) is a severe global cerebral dysfunction [1]. It is present in up to $70 \%$ and is associated with an increase in mortality due to sepsis $[1,2]$. An unspecific psychosyndrome with alterations of consciousness is the main clinicalblank;symptom. In most of the cases its clinical appearance precedes other symptoms of general inflammation [2-4]. Perimicrovascular edema and bleeding, endothelial activation, neuronal apoptosis, glial activation, ion channel differences, synthesis of the inducible nitricoxide synthase (iNOS) and changes in neurotransmitter release or concentrations have been reported $[1-3,5,6]$. Nevertheless, its pathophysiology is poorly understood andblank; little is known about gene expression differences within the brain and its compartments during sepsis-related encephalopathy.

In recent years the concept of early microcirculatory dysfunction as a motor of sepsis-related organ failure gained some attraction. Interestingly, microcirculatory dysfunction and inappropriate blood supply of organ cells on a microscopic level occur when overall blood flow and blood pressure (BP) are still in the normal range. This might explain, why studies on the overall cerebral blood flow did not find a relation to early sepsis-associated delirium $[7,8]$. However, by preventing excessive NO- 
production selective iNOS inhibitors attenuate the vascular dysfunction in a sepsis syndrome such as hypotension, endothelial dysfunction and microvascular shunting [9, 10]. They may additionally have immunomodulatory properties which might improve outcome [11].

In this study we aimed to find candidate genes associated with sepsis-associated delirium. As gene expression alterations occur early in the course of sepsis we required a standardized method concerning time and severity and chose again an endotoxic shock model. We analyzed gene expression differences of rat brain homogenate on microarrays at the time points of primarily recognized changes in the microvascular $(1 \mathrm{~h})$ and neuronal dysfunction $(3 \mathrm{~h})$ and finally $4.5 \mathrm{~h}$ after LPS administration before BP reached the critical lower level of cerebral autoregulation [12]. For validation a subset of genes was confirmed by real-time PCR. Additionally some candidate genes were studied in different brain compartments which are associated with clinical alterations in sepsis-related encephalopathy (hippocampus, somatosensory cortex, cerebellum).

To address the effect of selective iNOS inhibition in more detail we investigated the genetic effects of $1,400 \mathrm{~W}$ ( $N$-[3-(aminomethyl)benzyl]acetamide) in the septic brain.

Norepinephrine prevents the hemodynamic breakdown during sepsis and belongs to the classic therapeutic strategies. We studied whether rats being treated with LPS and norepinephrine showed mRNA expression differences within genes of interest found in the microarray studies.

\section{Materials and methods}

Rat model of sepsis

All procedures performed on the animals were in strict accordance with the National Institutes of Health Guide for Care and Use of Laboratory Animals and approved by the local Animal Care and Use Committee. The study was approved by the Institutional Review Board for the care of animal subjects.

Sprague Dawley rats (290-320 g) were initially anesthetized with isoflurane, tracheotomized, paralyzed with pancuronium bromide $[0.2 \mathrm{mg} /(\mathrm{kg} \mathrm{h})]$ and artificially ventilated (Harvard Rodent Ventilator; Harvard, South Natick, MA, USA). The right femoral artery and vein were cannulated for BP recording, blood sampling and drug administration. Rectal body temperature was maintained at $37^{\circ} \mathrm{C}$ using a feedback-controlled heating pad.

Approximately $60 \mathrm{~min}$ before LPS/placebo administration, isoflurane anesthesia was discontinued and replaced by an intravenous application of $\alpha$-chloralose ( $80 \mathrm{mg} / \mathrm{kg}$; Sigma-Aldrich Chemie GmbH, Taufkirchen, Germany). Supplementary doses of chloralose (30 mg/kg) were given every hour. During chloralose anesthesia, the animals were ventilated with a 1:1 mixture of nitrogen and oxygen. Arterial blood gas analyses and $\mathrm{pH}$ were measured repeatedly as needed and at least every $30 \mathrm{~min}$ (Blood gas analyzer model Rapidlab 348, Bayer Vital $\mathrm{GmbH}$, Fernwald, Germany). Also glucose and lactate levels were measured repeatedly (Glukometer Elite XL, Bayer Vital GmbH, Fernwald, Germany; Lactate pro, Arkray Inc. European Office, Düsseldorf, Germany). Glucose levels were kept at $>60 \mathrm{mg} / \mathrm{dl}$.

In random order 54 rats intravenously received $5 \mathrm{mg} /$ kg body weight LPS (from Escherichia coli, O111:B4, Sigma-Aldrich Chemie $\mathrm{GmbH}$, Germany) or $0.5 \mathrm{ml}$ of $0.9 \% \mathrm{NaCl}$. LPS was solved in $0.5 \mathrm{ml}$ of $0.9 \% \mathrm{NaCl}$ and slowly given within $5 \mathrm{~min}$. A moderate volume therapy of $1.2 \mathrm{ml} / \mathrm{h}$ of $0.9 \% \mathrm{NaCl}$ was given to replace renal and perspirative fluid losses. Septic rats and sham controls were killed 1,3 or $4.5 \mathrm{~h}$ after LPS or $\mathrm{NaCl}$ administration. Each experimental group consisted of nine animals.

Additionally, 18 rats received norepinephrine (Aventis GmbH, Bad Soden, Germany) or 1,400 W (Alexis Corporation, San Diego, USA) intravenously, beginning $1 \mathrm{~h}$ after LPS infusion. About $1,400 \mathrm{~W}$ was primarily applied as a bolus $(2.6 \mathrm{mg} / \mathrm{kg})$, followed by a continuous infusion $(2.6 \mathrm{mg} / \mathrm{kg} \times \mathrm{h})$. This dose was known to improve mean systemic arterial blood pressure (SAP) in the range of $80-90 \mathrm{mmHg}$. To achieve restoration of SAP accordingly continuous infusion of norepinephrine was titrated $(0.01-10 \mu \mathrm{g} / \mathrm{kg} \times \mathrm{min})$. Brains were removed and stored at $-80^{\circ} \mathrm{C}$.

\section{RNA isolation}

Cryosections $(10 \mu \mathrm{m})$ of whole rat brain or brain compartments were mixed with $1 \mathrm{ml}$ TriFast (PeqGOLD, peqlab, Erlangen, Germany) and homogenized using a $20 \mathrm{G}$ needle. After addition of $200 \mu \mathrm{l}$ chloroform (SigmaAldrich, Steinhelm, Germany), mixing, $10 \mathrm{~min}$ incubation at $4^{\circ} \mathrm{C}$ and centrifugation $\left(4^{\circ} \mathrm{C}, 20,000 \mathrm{~g}, 15 \mathrm{~min}\right)$, the upper phase was transferred into a new vial. After addition the same volume of $70 \%$ ethanol, RNA was isolated using the RNeasy Mini Kit (Qiagen, Hilden, Germany) following the manufacturer's instructions including a DNase digestion (RNase-free DNase Set, Qiagen). RNA was eluted in $34 \mu \mathrm{l}$ of RNase-free water, and quality was assessed by capillary electrophoresis using the Bioanalyzer 2100 (Agilent Technologies, Palo Alto, CA).

\section{Microarray experiments}

For labeling reactions $1 \mu \mathrm{g}$ of pooled total RNA $(n=3$ animals/pool) was used. Sample preparation (reverse transcription, T7-RNA amplification, labeling, purification, hybridization, washing and drying of the slides) was 
performed according to the Two-Color Microarray-Based Gene Expression Analysis Protocol (Version 5.5) using the RNA Spike-In Kit, the Low RNA Input Linear Amp Kit and the Cyanine CTP2-color dye pack (Agilent Technologies, Wilmington, USA).

Samples were alternatingly labeled using either $\mathrm{Cy} 3$ or Cy5 to match a balanced dye-swap design. Labeled aRNAs were hybridized overnight to 60mer oligonucleotide-spotted microarray slides (Rat Whole Genome $4 \times 44 \mathrm{~K}$; Agilent Technologies).

The dried slides were scanned using a GenePix 4100A scanner (Axon Instruments, Downingtown, PA). Image analysis was performed using the GenePix Pro 5.0 software, and calculated values for all spots were saved as GenePix result files. Stored data were evaluated using the $\mathrm{R}$ software and the limma package from BioConductor [13-15]. The spots were weighted for subsequent analyses according to the spot intensity, homogeneity and saturation. Spot intensities were corrected for the local background using the method of Edwards [16] with an offset of 64 to stabilize the variance of low-intensity spots. The M/A data were LOESS-normalized [17] before averaging. Genes were ranked for differential expression using a moderated $t$-statistic [18]. $P$ values were adjusted for multiple testing using the procedure of Benjamini and Hochberg [19]. Candidate lists were created by adjusting the false-discovery rate to $10 \%$. Afterwards, some of the genes found to be regulated were checked for their differential expression in the whole time course. We compared gene expression differences of following groups: rats killed 1, 3 and $4.5 \mathrm{~h}$ after LPS versus $\mathrm{NaCl}$ application and $4.5 \mathrm{~h}$ after LPS versus LPS $+1,400 \mathrm{~W}$ (3 repetitions each).

\section{Real-time PCR}

To confirm the results obtained by microarray experiments, the regulation of a subset of genes was analyzed by real-time PCR using the $\Delta \Delta \mathrm{C}_{\mathrm{T}}$ method for the calculation of relative changes [20]. Means and confidence intervals were calculated from the $\Delta \Delta \mathrm{C}_{\mathrm{T}}$ data. $P$ values were calculated using a two-sided $t$-test.

Within some genes of interest we also analyzed expression profiles in selected compartments. Differences of the fold-expressions between the compartments (Fig. 2) were analyzed by ANOVA and Dunnett's multiple-to-one comparisons against homogenate (when $P<0.05$ ). The logarithms of the fold-expressions were used to obtain normal distributed residuals. Normal distribution was confirmed with Shapiro-Wilk test, homogeneity of variances between groups was confirmed with Levene's test.

Per reaction, we used $1 \mu \mathrm{g}$ of a total RNA. Real-time PCR was performed using the 7900HT Fast Real-Time PCR system (Applied Biosystems, Foster City, USA). Porphobilinogen deaminase (PBGD) was used as reference gene. For cDNA synthesis, reagents and incubation steps were applied as described previously [21]. PCR reactions were set up using the Platinum SYBR Green qPCR SuperMix-UDG (Invitrogen, Karlsruhe, Germany). Sequences of the oligonucleotide primer pairs are given in Table 1 (final concentration $200 \mathrm{nM}$ ). Cycling conditions were $95^{\circ} \mathrm{C}$ for $6 \mathrm{~min}$, followed by 45 cycles of $95^{\circ} \mathrm{C}$ for $5 \mathrm{~s}, 59^{\circ} \mathrm{C}$ for $5 \mathrm{~s}$ and $72^{\circ} \mathrm{C}$ for $15 \mathrm{~s}$. For every gene we performed three independent experiments comparing pools of three animals each (performing double measurements). Due to the non-selective dsDNA binding of

Table 1 Primer sequences and amplicon sizes

\begin{tabular}{|c|c|c|c|c|}
\hline \multirow[t]{2}{*}{ Gene } & \multirow{2}{*}{$\begin{array}{l}\text { Gene } \\
\text { bank } \\
\text { accession }\end{array}$} & \multicolumn{2}{|l|}{ Primer sequence $\left(5^{\prime} \rightarrow 3^{\prime}\right)$} & \multirow{2}{*}{$\begin{array}{l}\text { Amplicon } \\
\text { length } \\
\text { (bp) }\end{array}$} \\
\hline & & Forward primer & Reverse primer & \\
\hline $\begin{array}{l}\text { Porphobilinogen deaminase } \\
\text { (PBGD) }\end{array}$ & 25709 & CAAGGTTTTCAGCATCGCTACCA & ATGTCCGGTAACGGCGGC & 135 \\
\hline $\begin{array}{l}\text { Baculoviral IAP repeat-containing } 3 \\
\text { (Birc3) }\end{array}$ & 78971 & TGGTCAGCAAAGTTCCGTAGG & AGGAAGCAGCCTTTCTCC & 119 \\
\hline $\begin{array}{l}\text { Chemokine ( } \mathrm{C}-\mathrm{C} \text { motif) ligand } 2 \\
(\mathrm{Ccl} 2)\end{array}$ & 24770 & CATCCACGTGCTGTCTCAGC & TCTCCAGCCGACTCATTGG & 95 \\
\hline $\begin{array}{l}\text { Chemokine }(\mathrm{C}-\mathrm{C} \text { motif) ligand } 20 \\
(\mathrm{Cc} 20)\end{array}$ & 29538 & TTCCTGGAGCTGAGAATGGC & GTGTACGTGAGGCAGCAGTCA & 122 \\
\hline $\begin{array}{l}\text { Chemokine (C-X-C motif) ligand } 1 \\
\text { (Cxcl1) }\end{array}$ & 81503 & GCCCCCATGGTTCAGAAGA & GAAGCCAGCGTTCACCAGA & 108 \\
\hline $\begin{array}{l}\text { Chemokine (C-X-C motif) ligand } 2 \\
(\mathrm{Cxcl} 2)\end{array}$ & 114105 & CCTTGGTTCAGAGGATCGTCC & GATTCTGCCCGTTGAGGTACA & 99 \\
\hline $\begin{array}{l}\text { Chemokine (C-X-C motif) ligand } 10 \\
\text { (Cxcl10) }\end{array}$ & 245920 & GTCGTTCTCTGCCTCGTGCT & AGTTTTCCTATGGCCCTGGG & 125 \\
\hline $\begin{array}{l}\text { Endothelial cell-specific molecule } 1 \\
\text { (Esm1) }\end{array}$ & 64536 & CATATGTGACAGGGTGACCGG & TCTCACAGCATTGCCATCTCC & 133 \\
\hline Lipocalin 2 (Lcn2) & 170496 & ACAACGTCACTTCCATCCTCG & CCTGGAGCTTGGAACGAATG & 74 \\
\hline Reelin (Reln) & 24718 & AAAGGCCAGCACCCGTTAC & GCCCGAGATCCACGGAGTA & 140 \\
\hline
\end{tabular}

Sequences were taken from Gene bank, accession numbers are given 


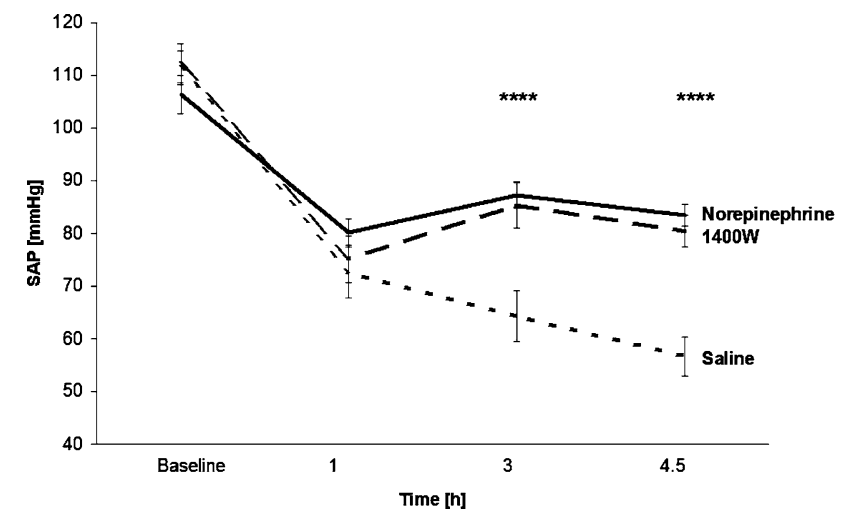

Fig. 1 Influence of norepinephrine and 1,400 W onto the mean systemic arterial blood pressure (SAP) after LPS application. Mean \pm SEM of nine independent experiments each; $* * * * P<0.0001$ versus LPS/saline-treated animals. SAP was significantly increased in both therapy groups

the SYBR Green I dye, melting curve analysis and gel electrophoresis were performed.

\section{Western blot}

We performed SDS-PAGE with $15 \%$ polyacrylamide gels (stacking gel 5\%) using the BioRad tank blot system (BioRad, Munich, Germany). After incubation with Laemmli buffer $\left(5 \mathrm{~min} / 95^{\circ} \mathrm{C}\right)$, running the gel, blotting and $2 \mathrm{~h}$ of blocking (5\% fat-free, skimmed milk/PBS), primary antibodies (detecting Cxcl1 and $\mathrm{Cxcl} 2$, US Biological, Swampscott, MA, USA) were incubated overnight (rotating $4^{\circ} \mathrm{C}$ ) and secondary antibodies (labeled with horseradish peroxidase) for $1 \mathrm{~h}$ (room temperature, moving). For detection of bands we used the
Amersham ECL Plus Western Blotting Detection Reagents (GE Healthcare, Munich, Germany).

Immunofluorescence staining

Paraffin sections $(10 \mu \mathrm{m})$ of septic brain were deparaffinized and rehydrated using xylol and a series of ethanol in PBS dilutions. Antigen retrieval was performed in a microwave $(630 \mathrm{~W} / 7 \mathrm{~min})$ with PBS/1 mM EDTA.

After cooling to room temperature, sections were blocked with 5\% BSA in PBS. Primary (1:100) and secondary antibodies $(1: 1,000$, both in PBS/ $0.1 \%$ BSA) were hybridized for $1 \mathrm{~h}$ at room temperature. After each binding step, we washed with PBS/0.1\% BSA. Nuclei were stained with DAPI $(1 \mu \mathrm{mol} / \mathrm{ml}, 5 \mathrm{~min}$; AppliChem, Darmstadt, Germany). Final fixation was performed with $4 \%$ paraformaldehyde (10 $\mathrm{min})$.

\section{Results}

No rat died from LPS injection. As expected, rats developed a severe sepsis syndrome with significant drop in BP (Fig. 1) and pH and increase in lactate levels (Table 2). The higher $\mathrm{pH}$ during baseline was related to chloralose narcosis leading to metabolic alcalose [22]. $\mathrm{pH}$ decreased approximately by 0.1 in all groups till end of experiments. According to a transient hypercapnia in the norepinephrine group $\mathrm{pH}$-levels were lower at 1 and $3 \mathrm{~h} .1,400 \mathrm{~W}$ and norepinephrine stabilized BP in the lower normal range (Fig. 1). Only norephinephrine resulted in a lower lactate level at the end of experiments (Table 2).
Table 2 Arterial blood gases and lactate in rats with LPSinduced septic shock in response to norepinephrine and $1,400 \mathrm{~W}$

\begin{tabular}{lllll}
\hline Time after LPS (min) & 0 & 60 & 180 & 270 \\
\hline $\mathrm{PO}_{2}$ & & & & \\
LPS & $265.8 \pm 5.8$ & $263.7 \pm 4.5$ & $273.9 \pm 2.7$ & $269.5 \pm 4.6$ \\
LPS + norepinephrine & $279.6 \pm 5.8$ & $265.8 \pm 6.1$ & $263.1 \pm 5.1$ & $267.7 \pm 4.9$ \\
LPS + 1,400 W & $264.0 \pm 10.1$ & $264.6 \pm 9.6$ & $259.8 \pm 9.7$ & $253.3 \pm 17.3$ \\
$\mathrm{PCO}_{2}$ & & & \\
LPS & $31.3 \pm 1.0$ & $34.7 \pm 0.8$ & $33.5 \pm 0.7$ & $32.3 \pm 0.6$ \\
LPS + norepinephrine & $30.2 \pm 0.5$ & $39.8 \pm 1.6 * * *$ & $39.3 \pm 1.2^{* * *}$ & $32.8 \pm 0.8$ \\
LPS + 1,400 W & $31.3 \pm 0.5$ & $33.2 \pm 1.5$ & $34.6 \pm 2.3$ & $32.6 \pm 0.9$ \\
pH & & & \\
LPS & $7.56 \pm 0.01$ & $7.43 \pm 0.01$ & $7.45 \pm 0.01$ & $7.45 \pm 0.01$ \\
LPS + norepinephrine & $7.57 \pm 0.01$ & $7.40 \pm 0.02^{* *}$ & $7.38 \pm 0.02^{* * *}$ & $7.46 \pm 0.02$ \\
LPS + 1,400 W & $7.57 \pm 0.01$ & $7.43 \pm 0.02$ & $7.42 \pm 0.02$ & $7.45 \pm 0.01$ \\
Lactate & & & \\
LPS & $0.94 \pm 0.17$ & $2.57 \pm 0.23$ & $2.26 \pm 0.10$ & $2.37 \pm 0.23$ \\
LPS + norepinephrine & $0.85 \pm 0.20$ & $2.18 \pm 0.13$ & $2.09 \pm 0.20$ & $1.53 \pm 0.20^{* *}$ \\
LPS + 1,400 W & $0.82 \pm 0.16$ & $2.40 \pm 0.17$ & $2.34 \pm 0.13$ & $2.14 \pm 0.07$ \\
\hline
\end{tabular}

$\mathrm{PO}_{2}, \mathrm{PCO}_{2}$ and $\mathrm{pH}$ : whole arterial blood data (A. femoralis); lactate: arterial plasma data; $n=9$; mean \pm SEM

$* * P \leq 0.01, * * * P \leq 0.001$, rest: not significant 
Tables 3 and 4 show the group-averaged data of several candidate genes found in microarray studies. The most impressive regulations were observed in the group of chemokines and interleukines. Other genes were involved in coagulation, regulation of apoptosis, composition of extracellular matrix, metabolism, transcription and cell cycle regulation.

The 1,400 W therapy group showed hardly any gene expression differences compared to the intravenous LPS group after $4.5 \mathrm{~h}$ (Table 4, complete list given).

Within the group of PCR-validated genes 100\% were confirmed to be up- or down-regulated (Table 5). In the norepinephrine therapy group, real-time PCRs revealed a tendency of chemokine-induction compared to the LPS group (Table 5).

We additionally evaluated the expression level of several LPS-induced genes within the hippocampus, the somatosensory cortex and the cerebellum; Ccl2, Ccl20, Cxcl2 and endothelial cell-specific molecule 1 (Esm1) were found to be most intensively induced within the hippocampus. The other compartments also frequently showed a stronger gene regulation compared to brain homogenate, yet to a lower degree (Fig. 2).

Studying Cxcl1 and Cxcl2 expression in brain homogenate, we could confirm our RNA data on protein level. Western blots revealed an increased expression of

Table 3 Gene expression differences 1, 3 and $4.5 \mathrm{~h}$ after intravenous LPS administration (microarray data)

\begin{tabular}{|c|c|c|c|c|c|}
\hline \multirow[t]{2}{*}{ Group } & \multirow[t]{2}{*}{ Gene } & \multicolumn{4}{|c|}{ Ratio LPS/NaCl: mean \pm SEM } \\
\hline & & $\begin{array}{l}\text { Gene bank } \\
\text { accession }\end{array}$ & $1 \mathrm{~h}$ & $3 \mathrm{~h}$ & $4.5 \mathrm{~h}$ \\
\hline \multirow[t]{16}{*}{ Inflammation } & Interleukin 1 beta $(\mathrm{Il} 1 \beta)$ & NM_031512 & $4.04 \pm 0.37 *$ & $6.66 \pm 0.33 * * *$ & $9.23 \pm 0.34 * * *$ \\
\hline & Interleukin 6 (Il6) & NM_012589 & $1.26 \pm 0.33 \mathrm{NS}$ & $6.04 \pm 0.30 * * *$ & $6.65 \pm 0.30 * * *$ \\
\hline & $\begin{array}{l}\text { Chemokine (C-X-C motif) ligand } 1 \\
\text { (Cxcl1) }\end{array}$ & NM_030845 & $5.53 \pm 0.53 \mathrm{NS}$ & $11.92 \pm 0.48 * * *$ & $34.42 \pm 0.48 * * *$ \\
\hline & $\begin{array}{l}\text { Chemokine (C-X-C motif) ligand } 2 \\
(\mathrm{Cxcl} 2)\end{array}$ & NM_053647 & $5.22 \pm 0.40^{*}$ & $15.52 \pm 0.37 * * *$ & $33.43 \pm 0.37 * * *$ \\
\hline & $\begin{array}{l}\text { Chemokine (C-X-C motif) ligand } 9 \\
\text { (Cxc19) }\end{array}$ & NM_145672 & $0.86 \pm 0.40 \mathrm{NS}$ & $3.01 \pm 0.361^{*}$ & $4.26 \pm 0.37 * * *$ \\
\hline & $\begin{array}{l}\text { Chemokine (C-X-C motif) ligand } 10 \\
\text { (Cxcl10) }\end{array}$ & NM_139089 & $1.36 \pm 0.45 \mathrm{NS}$ & $15.74 \pm 0.41 * * *$ & $21.26 \pm 0.41 * * *$ \\
\hline & Chemokine $(\mathrm{C}-\mathrm{C}$ motif) ligand $2(\mathrm{Ccl} 2)$ & NM_031530 & $1.94 \pm 0.44 \mathrm{NS}$ & $26.76 \pm 0.40 * * *$ & $21.71 \pm 0.41 * * *$ \\
\hline & Chemokine $(\mathrm{C}-\mathrm{C}$ motif) ligand $3(\mathrm{Ccl} 3)$ & NM_013025 & $2.15 \pm 0.41 \mathrm{NS}$ & $3.65 \pm 0.371 * *$ & $4.45 \pm 0.38 * * *$ \\
\hline & Chemokine $(\mathrm{C}-\mathrm{C}$ motif) ligand 7 (Ccl7) & NM_001007612 & $1.22 \pm 0.278 \mathrm{NS}$ & $3.45 \pm 0.252 * * *$ & $4.39 \pm 0.26 * * *$ \\
\hline & Chemokine $(\mathrm{C}-\mathrm{C}$ motif) ligand $20(\mathrm{Ccl} 20)$ & NM_019233 & $0.99 \pm 0.37 \mathrm{NS}$ & $8.73 \pm 0.34 * * *$ & $9.80 \pm 0.343 * * *$ \\
\hline & $\begin{array}{l}\text { Interferon stimulated exonuclease } 20 \\
\text { (Isg20) }\end{array}$ & NM_001008510 & $1.10 \pm 0.41 \mathrm{NS}$ & $4.39 \pm 0.37 * *$ & $2.69 \pm 0.37 \mathrm{NS}$ \\
\hline & $\begin{array}{l}\text { Interferon-induced protein with } \\
\text { tetratricopeptide repeats } 2 \text { (Ifit } 2 \text { ) }\end{array}$ & NM_001024753 & $0.97 \pm 0.38 \mathrm{NS}$ & $4.05 \pm 0.34 * * *$ & $4.18 \pm 0.35 * * *$ \\
\hline & $\begin{array}{l}\text { Similar to interferon-inducible GTPase } \\
\text { (MGC108823) }\end{array}$ & NM_001012353 & $0.97 \pm 0.36 \mathrm{NS}$ & $5.20 \pm 0.33 * * *$ & $3.17 \pm 0.33 * *$ \\
\hline & Interferon-induced protein 44 (Ifi44) & XM_227821 & $1.27 \pm 0.32 \mathrm{NS}$ & $2.76 \pm 0.29 * *$ & $2.52 \pm 0.29 *$ \\
\hline & Interferon gamma induced GTPase (Igtp) & XM_220451 & $1.02 \pm 0.23 \mathrm{NS}$ & $2.37 \pm 0.21 * * *$ & $1.48 \pm 0.21 \mathrm{NS}$ \\
\hline & $\begin{array}{l}\text { Tumor necrosis factor receptor } \\
\text { superfamily, member } 5 \text { (Tnfrsf5) }\end{array}$ & NM_134360 & $1.15 \pm 0.28 \mathrm{NS}$ & $2.40 \pm 0.25^{* *}$ & $3.37 \pm 0.25 * * *$ \\
\hline \multirow[t]{2}{*}{ Coagulation } & Protein $\mathrm{C}$ receptor, endothelial (Procr) & NM_001025733 & $1.13 \pm 0.30 \mathrm{NS}$ & $2.90 \pm 0.28 * *$ & $3.89 \pm 0.28 * * *$ \\
\hline & Tissue factor pathway inhibitor 2 (Tfpi2) & NM_173141 & $1.10 \pm 0.39 \mathrm{NS}$ & $3.29 \pm 0.36^{*}$ & $7.45 \pm 0.36 * * *$ \\
\hline \multirow[t]{2}{*}{$\begin{array}{l}\text { Matrix } \\
\text { remodeling }\end{array}$} & $\begin{array}{l}\text { Tissue inhibitor of metalloproteinase } 1 \\
\text { (Timp1) }\end{array}$ & NM_053819 & $0.95 \pm 0.43 \mathrm{NS}$ & $3.33 \pm 0.39^{*}$ & $6.66 \pm 0.39 * * *$ \\
\hline & Hyaluronan synthase 1 (Has1) & NM_172323 & $1.21 \pm 0.34 \mathrm{NS}$ & $4.41 \pm 0.31 * * *$ & $5.18 \pm 0.32 * * *$ \\
\hline Anti-apoptotic & $\begin{array}{l}\text { Baculoviral IAP repeat-containing } 3 \\
\text { (Birc3) }\end{array}$ & NM_023987 & $1.24 \pm 0.28 \mathrm{NS}$ & $2.65 \pm 0.26^{* *}$ & $4.72 \pm 0.26 * * *$ \\
\hline Endothelium & $\begin{array}{l}\text { Endothelial cell-specific molecule } 1 \\
\text { (Esm1) }\end{array}$ & NM_022604 & $2.30 \pm 0.50 \mathrm{NS}$ & $3.40 \pm 0.45 \mathrm{NS}$ & $6.21 \pm 0.46 * * *$ \\
\hline Bacteriostatic & Lipocalin 2 (Lcn2) & NM_130741 & $1.06 \pm 0.37 \mathrm{NS}$ & $1.92 \pm 0.34 \mathrm{NS}$ & $3.15 \pm 0.34 *$ \\
\hline Kinase regulation & $\begin{array}{l}\text { Mitogen-activated protein kinase } \\
\text { kinase kinase } 8 \text { (Map3k8) }\end{array}$ & NM_053847 & $1.43 \pm 043 \mathrm{NS}$ & $4.73 \pm 0.39 * *$ & $2.91 \pm 0.39 \mathrm{NS}$ \\
\hline $\begin{array}{l}\text { Cell cycle } \\
\text { regulation }\end{array}$ & $\begin{array}{l}\text { Splicing factor, arginine/serine-rich } 5 \\
\text { (Sfrs5) }\end{array}$ & NM_019257 & $0.33 \pm 0.30 *$ & $0.56 \pm 0.27 \mathrm{NS}$ & $1.21 \pm 0.28 \mathrm{NS}$ \\
\hline
\end{tabular}

The strongest regulations in the time course are given in bold let- NS not significant ters. In most of the cases they were found after $4.5 \mathrm{~h}$. Expectedly, $*$ Adjusted $P \leq 0.05$; **adjusted $P \leq 0.01, * * *$ adjusted $P \leq 0.001$ the group of inflammation-associated genes was the largest and contained the strongest gene regulations 
Table 4 Gene expression differences $4.5 \mathrm{~h}$ after intravenous LPS administration, 1,400 W therapy group (microarray data)

\begin{tabular}{lll}
\hline Gene & $\begin{array}{l}\text { Gene bank } \\
\text { accession }\end{array}$ & $\begin{array}{l}\text { 1,400 W + LPS/LPS: } \\
\text { mean } \pm \text { SEM }\end{array}$ \\
\hline Inositol polyphosphate-4-phosphatase, type II (Inpp4b) & NM_053917 & $2.37 \pm 1.46$ NS \\
NADH dehydrogenase (ubiquinone) 1, subcomplex unknown, 2 (Ndufc2) & NM_001009290 & $2.09 \pm 0.41$ NS \\
Splicing factor, arginine/serine-rich 5 (Sfrs5) & NM_019257 & $0.49 \pm 0.28$ NS \\
Olfactomedin 3 (Olfm3) & NM_145777 & $0.45 \pm 0.41$ NS \\
Reelin (Reln) & NM_080394 & $0.38 \pm 0.51$ NS \\
Synaptic vesicle glycoprotein 2b (Sv2b) & NM_057207 & $0.21 \pm 0.36^{* * *}$
\end{tabular}

NS not significant

** Adjusted $P \leq 0.01$. Except for Sv2b, we found no significant gene expression differences in the 1,400 W therapy group compared to the LPS group

both molecules in the LPS-stimulated group (Fig. 3). In the hippocampal area we found a neuron-specific staining for Cxcl1 (positive reaction of the same cells with NeuN antibody, which specifically marks neurons). Additionally $\mathrm{Cxcl} 2$ was detected in axons of the white matter (Fig. 4).

\section{Discussion}

Early occurrence of gene expression alterations, induction of cytokine levels together with neuronal dysfunction point to a complex brain inflammatory response syndrome (BIRS), which precedes the sepsis-induced systemic response syndrome (SIRS). This concept is in line with the clinical observation of early sepsis-associated delirium as an indicator of a systemic sepsis syndrome [1-4]. Whereas modern therapeutic regimes mainly focus on the prevention from early microcirculatory dysfunction the immunomodulatory effects of iNOS inhibitors or catecholamines in the septic brain were still unknown.

Microarray data revealed a strong chemokine up-regulation after $1 \mathrm{~h}$, further increasing $3-4.5 \mathrm{~h}$ after intravenous LPS injection (Table 3). Results were confirmed by real-time PCR; we actually found a more pronounced regulation level using this method (Table 5), what is a common phenomenon as the dynamic range of microarrays is smaller than that of real-time PCR. This becomes obvious especially when studying strongly regulated genes. Additionally, preamplification methods tend to cause a data compression [23-25].

Cxcl1 and $\mathrm{Cxcl} 2$ were also proven to be up-regulated in the LPS group on protein level (Fig. 3), yet to a lower degree. Presumably, this discrepancy was caused by a delayed induction of gene expression on the protein level.

Chemokines play an important role in the recruitment and regulation of leukocyte traffic during bacterial infection [26]. The $\mathrm{Ccl} 2$ (macrophage chemoattractant protein1, MCP-1) serum level in acute peritonitis was greatest in patients who died [27]. Consistently, patients with severe forms of sepsis revealed highest Ccl2 plasma levels [28]. Low Ccl2 plasma levels are associated with survival in invasive pneumococcal disease and correlate with bacterial loads in blood and cerebrospinal fluid, disease presentation and outcome [26]. Intrahippocampal injections of $\mathrm{Ccl} 2$ or $\mathrm{Cxcl} 2$ (macrophage inflammatory protein-2, MIP-2) resulted in the apoptosis of hippocampal neurons, thus supporting a direct role of these chemokines in neuronal death [29]. Yet chemokine-activated astrocytes seemed to

Table 5 Validations using real-time PCR: Gene expressions in brain homogenate $4.5 \mathrm{~h}$ after administration of LPS or LPS + treatment $(1,400 \mathrm{~W} /$ norepinephrine)

\begin{tabular}{lllll}
\hline Gene & $\begin{array}{l}\text { Array LPS/control: } \\
\text { mean } \pm \text { SEM }\end{array}$ & $l$ PCR & \\
\cline { 3 - 5 } & & $\begin{array}{l}\text { LPS/control: } \\
\text { mean }(95 \% \mathrm{CI})\end{array}$ & $\begin{array}{l}\text { 1,400 W/LPS: } \\
\text { mean (95\% CI) }\end{array}$ & $\begin{array}{l}\text { Norepinephrine/LPS: } \\
\text { mean }(95 \% \text { CI })\end{array}$ \\
\hline Ccl2 & $21.71 \pm 0.41$ & $40.53(14.74: 111.43)^{* *}$ & $0.77(0.35: 1.69) \mathrm{NS}$ & $1.97(0.29: 13.34) \mathrm{NS}$ \\
Ccl20 & $9.80 \pm 0.34$ & $167.09(63.58: 439.11)^{* *}$ & $1.64(0.45: 6.00) \mathrm{NS}$ & $2.58(0.08: 81.08) \mathrm{NS}$ \\
Cxcl1 & $34.42 \pm 0.48$ & $256.51(57.03: 1,153.74)^{* *}$ & $1.72(1.37: 2.16)^{* *}$ & $1.77(0.36: 8.66) \mathrm{NS}$ \\
Cxcl2 & $33.43 \pm 0.37$ & $58.59(38.10: 90.12)^{* * * *}$ & $1.70(1.17: 2.46)^{*}$ & $2.60(0.96: 7.06) \mathrm{NS}$ \\
Cxcl10 & $21.26 \pm 0.41$ & $38.52(0.22: 6,897.33) \mathrm{NS}$ & $0.79(0.14: 4.62) \mathrm{NS}$ & $1.30(0.26: 6.37) \mathrm{NS}$ \\
Birc3 & $4.72 \pm 0.26$ & $6.72(3.54: 12.75)^{* *}$ & $6.75(0.25: 2.25) \mathrm{NS}$ & $0.73(0.23: 2.30) \mathrm{NS}$ \\
Esm1 & $6.21 \pm 0.46$ & $7.93(2.42: 25.96)^{*}$ & $0.78(0.08: 7.85) \mathrm{NS}$ & $0.78(0.31: 1.96) \mathrm{NS}$ \\
Lcn2 & $3.15 \pm 0.34$ & $5.37(1.41: 20.45)^{*}$ & $0.52(0.18: 1.50) \mathrm{NS}$ & $0.83(0.60: 1.14) \mathrm{NS}$ \\
\hline
\end{tabular}

$N S$ not significant, $C I$ confidence interval

Array data are those of Table 3 (Lcn2: $P=0.0102$, rest: $P \leq 0.001$ ). PCR data: three pools of three animals each were compared to same-sized control pools performing double measurements. $* P \leq 0.05, * * P \leq 0.01, * * * P \leq 0.001$ 

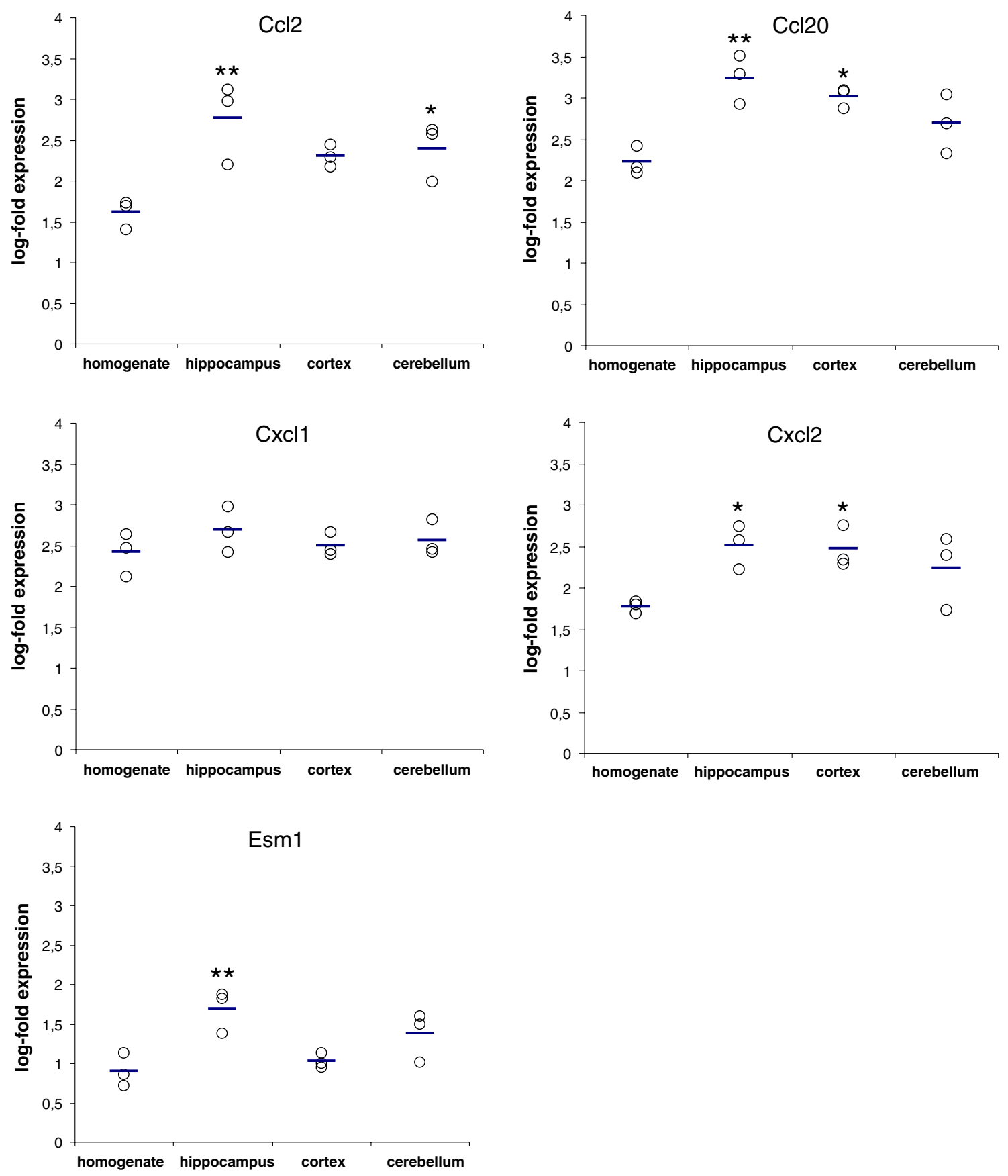

Fig. 2 LPS-induced changes of mRNA expression in brain homogenate and three compartments. $Y$-axis represent log fold mRNA expressions of LPS-treated compared to $\mathrm{NaCl}$-treated animals after $4.5 \mathrm{~h}$. Every spot represents a comparison of a $\mathrm{NaCl}$

promote the survival and differentiation of hippocampal neurons [29, 30]. Ccl2 via its receptor CCR2 plays also an important role in regulating the permeability of the bloodbrain-barrier which is reduced in CCR2 $2^{(-1-)}$ mice being subjected to focal transient cerebral ischemia and which was correlated with reduced infarct sizes [31]. However,

pool with an LPS pool (3 animals/pool). Horizontal lines indicate the geometric mean. Chemokines and Esm1 were up-regulated, especially within the hippocampus. ${ }^{*} P \leq 0.05, * * P \leq 0.01$

we did not find significant brain edema formation in our rat model up to $4.5 \mathrm{~h}$ (unpublished data).

Since gene expression differences in the $1,400 \mathrm{~W}$ group were only subtle as compared to controls in the early phase of a sepsis syndrome significant immunomodulatory effects appear to be unlikely (Tables 4, 5). 


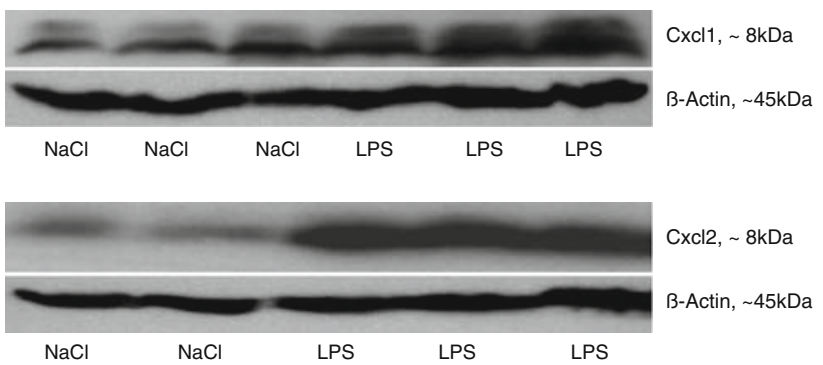

Fig. $3 \mathrm{Cxcl} 1$ and $\mathrm{Cxcl} 2$ protein expression in brain homogenate. The LPS-induced up-regulation of mRNA could be validated on the protein level $(4.5 \mathrm{~h}$ after $\mathrm{LPS} / \mathrm{NaCl})$

Therefore, our data support the notion of iNOS inhibitors to be mainly vasoprotective agents improving the outcome in a sepsis syndrome.

Norepinephrine seems to up-regulate the expression of $\mathrm{Ccl} 2$, Ccl20, Cxcl1 and Cxcl2 (Table 5), what suggests a possible proinflammatory potential of norepinephrine. Recent experiments on endotoxin-induced lung inflammation rendered a similar result, as phenylephrin increased the formation of $\mathrm{TNF} \alpha$ - and $\mathrm{Cxcl} 2$-specific mRNA by lung neutrophils [32]. Other groups found that norepinephrine exhibits proinflammatory properties onto the systemic TNF $\alpha$ - and IL1 $\beta$-release [33]. Tsuda et al. [34] found an impairment of the host's antibacterial resistance by norepinephrine-activated neutrophils. Additionally, the noradrenergic reuptake inhibitor desipramine augments the stress-induced IL1 $\beta$-response in the hippocampus [35]. Nevertheless, the norepinephrine therapy is still regarded as the most effective agent in treating sepsis-related hypodynamic failure.

Neither 1,400 W nor norepinephrine had a significant effect on the gene expression of Birc3 and Esm1, which were both induced by LPS (Table 3,5). Birc3 is necessary for the maintenance of a normal, infection-triggered innate immune response. Birc $3^{-1-}$ mice exhibit profound resistance to LPS-induced sepsis, specifically because of an attenuated inflammatory response [36]. Esm1 is a novel endothelial cell dysfunction marker. Septic rats showed an elevated Esm1 plasma level $4 \mathrm{~h}$ after LPS administration [37]. In septic patients the Esm1 blood level is related to the severity of illness and the outcome of the patient [38]. Therefore we suspect that Esm1 and Birc3 are two important genes that could be used as markers in therapeutic sepsis approaches. Interestingly, Esm1-specific mRNA was most intensively up-regulated in the LPS-stimulated hippocampus (Fig. 2), what forebodes a stronger susceptibility of hippocampal capillaries.

Comparing the chemokine ( $\mathrm{Ccl} 2, \mathrm{Ccl} 20, \mathrm{Cxcl} 2)$ expression levels of brain homogenate to those of the hippocampus, the somatosensory cortex and the cerebellum, we found a stronger LPS-effect in the selected compartments (Fig. 2), in which the hippocampus appeared to be most intensively stimulated. Consistent with our findings it appears that the hippocampus is a highly vulnerable brain region during experimental sepsis [39]. These data are in line with the clinical observation of delirium being an essential symptom of the sepsisassociated cerebral dysfunction.
Fig. 4 Localization of target genes. In immunofluorescence staining of LPS-treated brains, Cxcl1 accumulated in hippocampal neurons as proved by the neuron marker NeuN. $\mathrm{Cxcl} 2$ was primarily located within the axones of the white matter. Neu/Cxcl1 $\times 100, \mathrm{Cxcl} 2$ $\times 200$; blue DAPI, red target proteins

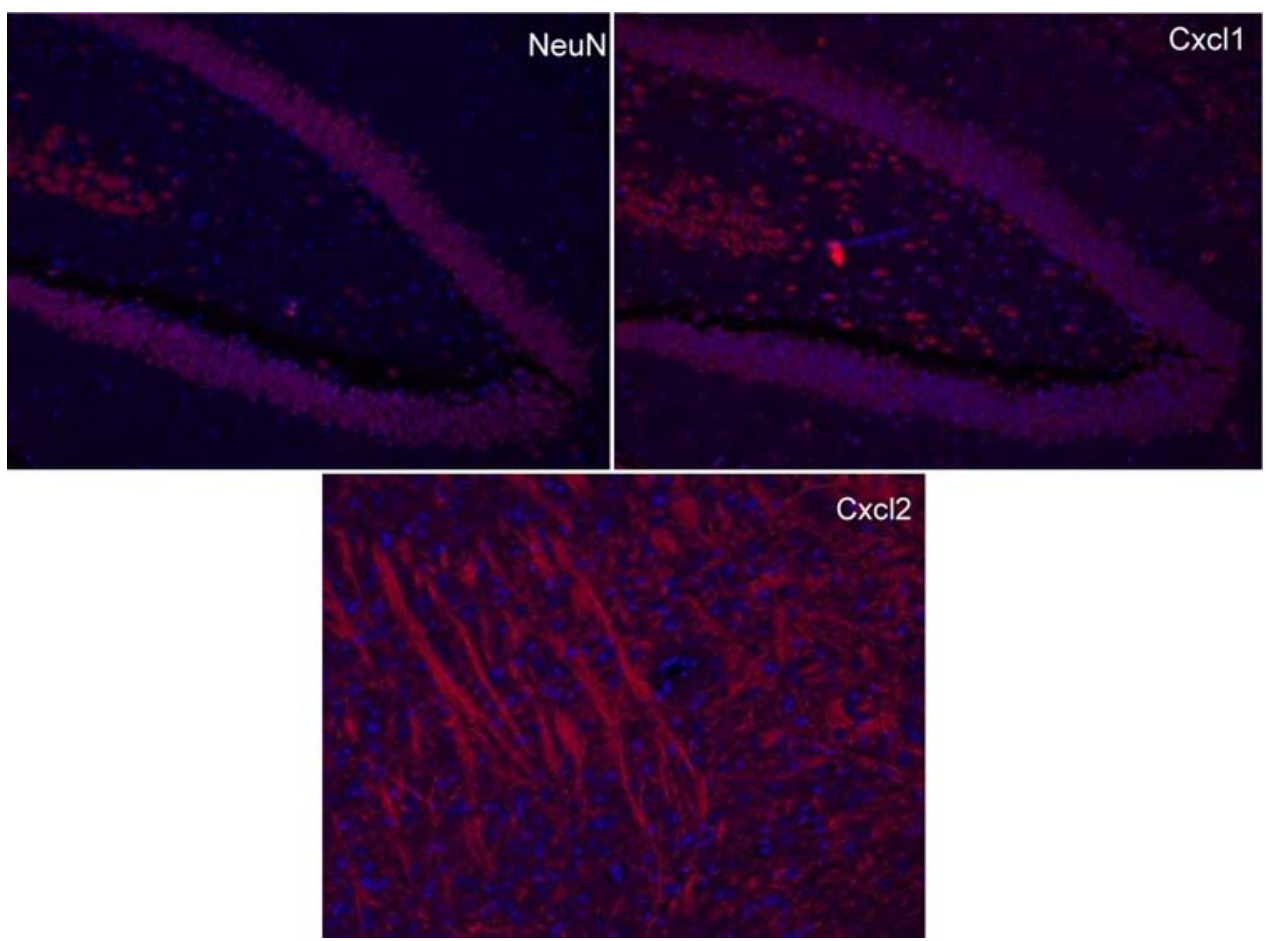


However, a limitation of the technique is that brain homogenate studies were not able to differentiate between glial cells and neurons. According to our own experience, laser-microdissection techniques were not suitable because of the complex shape of neurons and glial cells preventing isolation free of contaminations due to interfering cell processes. Comparing different brain compartments, the strong differences in gene expression underline the role of neurons and glial cells and cannot be related to blood vessel or blood cell effects. Accordingly, immunofluorescence staining of $\mathrm{Cxcl} 1$ presented a similar picture as NeuN with an accumulation in hippocampal neurons (Fig. 4). Cxcl2 showed an accumulation within the axones of the white matter.

The comparatively strong chemokine induction in the hippocampus and to a lower degree in the somatosensory cortex and the cerebellum could be explained by a pronounced breakdown of the blood-brain-barrier in these compartments, a stronger activation of astrocytes and an increased production within resident neurons. As the hippocampus seems to be the most susceptible region, further therapeutic approaches should include this compartment.

\section{Conclusions}

After LPS application, Esm1, Ccl2, Ccl20 and Cxcl2 were found to be most intensively up-regulated within the hippocampus. Hence, we assume that in sepsis-associated delirium the hippocampus is a compartment with high vulnerability. This is in line with the clinical observation of confusion and disturbance of memory. Birc3 and Esm1 may be used as prognostic parameters in further therapeutic approaches. Norepinephrine and 1,400 W seem to have no significant beneficial effect onto mRNA expression. Therefore, our data proved for the first time the assumption of a mainly vasoprotective mechanism of iNOS inhibitors in a sepsis syndrome.

Acknowledgments We thank Maria Magdalena Stein for skillful technical assistance. This study was supported by a start-up financing of the Justus-Liebig-University of Giessen, Germany.

Open Access This article is distributed under the terms of the Creative Commons Attribution Noncommercial License which permits any noncommercial use, distribution, and reproduction in any medium, provided the original author(s) and source are credited.

\section{References}

1. Ebersoldt M, Sharshar T, Annane D (2007) Sepsis-associated delirium. Intensive Care Med 33:941-950

2. Green R, Scott LK, Minagar A, Conrad S (2004) Sepsis associated encephalopathy (SAE): a review. Front Biosci 9:1637-1641

3. Papadopoulos MC, Davies DC, Moss RF, Tighe D, Bennett ED (2000) Pathophysiology of septic encephalopathy: a review. Crit Care Med 28:3019-3024

4. Bello JH, Park M (2007) Sepsisassociated encephalopathy as a differential diagnosis with motor deficit plus altered mental status. Clinics 62:199-202

5. Eggers V, Schilling A, Kox WJ, Spies C (2003) Septic encephalopathy. Diagnosis und therapy. Anaesthesist 52:294-303

6. Sharshar T, Annane D, de la Grandmaison GL, Brouland JP, Hopkinson NS, Françoise G (2004) The neuropathology of septic shock. Brain Pathol 14:21-33

7. Hinkelbein J, Schroeck H, Peterka A, Schubert C, Kuschinsky W, Kalenka A (2007) Local cerebral blood flow is preserved in sepsis. Curr Neurovasc Res 4:39-47
8. Pfister D, Siegemund M, Dell-Kuster S, Smielewski P, Rüegg S, Strebel SP, Marsch SC, Pargger H, Steiner LA (2008) Cerebral perfusion in sepsisassociated delirium. Crit Care 12:R63

9. Lee CC, Lin NT, Hsu YH, Chen HI (2005) Inducible nitric oxide synthase inhibition potentiates multiple organ dysfunction induced by endotoxin in conscious rats. J Cardiovasc Pharmacol 9:139-143

10. Matejovic M, Krouzecky A, Radej J, Rokyta R Jr, Kralova H, Radermacher P, Novak I (2007) Coagulation and endothelial dysfunction during longterm hyperdynamic porcine bacteremia-effects of selective inducible nitric oxide synthase inhibition. Thromb Haemost 97:304309

11. Ahn KS, Noh EJ, Zhao HL, Jung SH, Kang SS, Kim YS (2005) Inhibition of inducible nitric oxide synthase and cyclooxygenase II by Platycodon grandiflorum saponins via suppression of nuclear factor-kappaB activation in RAW 264.7 cells. Life Sci 76:23152328
12. Rosengarten B, Hecht M, Auch D, Ghofrani HA, Schermuly RT, Grimminger F, Kaps M (2007) Microcirculatory dysfunction in the brain precedes changes in evoked potentials in endotoxin-induced sepsis syndrome in rats. Cerebrovasc Dis 23:140-147

13. R Development Core Team (2007) R: a language and environment for statistical computing. R Foundation for statistical computing, Vienna, Austria. ISBN 3900051-07-0. Available via DIALOG http://www.R-project.org. Accessed 2007

14. Smyth GK (2005) Limma: linear models for microarray data. In: Gentleman R, Carey V, Dudoit S, Irizarry R, Huber W (eds) Bioinformatics and computational biology solutions using $\mathrm{R}$ and bioconductor. Springer, New York, pp 397-420 
15. Gentleman RC, Carey VJ, Bates DM, Bolstad B, Dettling M, Dudoit S, Ellis B, Gautier L, Ge Y, Gentry J, Hornik K, Hothorn $\mathrm{T}$, Huber $\mathrm{W}$, Iacus $\mathrm{S}$, Irizarry R, Leisch F, Li C, Maechler M, Rossini AJ, Sawitzki G, Smith C, Smyth G, Tierney L, Yang JY, Zhang J (2004) Bioconductor: open software development for computational biology and bioinformatics. In: Genome Biol 5:R80. Available via DIALOG http://genomebiology.com/2004/5/10/ R80. Accessed 2004

16. Edwards D (2003) Non-linear normalization and background correction in one-channel cDNA microarray studies. Bioinformatics 19:825-833

17. Smyth GK, Speed TP (2003) Normalization of cDNA microarray data. Methods 31:265-273

18. Smyth GK (2004) Linear models and empirical Bayes methods for assessing differential expression in microarray experiments. In: Statistical applications in genetics and molecular biology 3 , article 3. Available via DIALOG http://www.bepress.com/sagmb/vol3/ iss1/art3. Accessed 2004

19. Benjamini Y, Hochberg Y (1995) Controlling the false discovery rate: a practical and powerful approach to multiple testing. J R Stat Soc Ser B 57:289-300

20. Livak KJ, Schmittgen TD (2001) Analysis of relative gene expression data using real-time quantitative PCR and the 2(-Delta Delta C(T)) method. Methods 25:402-408

21. Fink L, Kohlhoff S, Stein MM, Hänze J, Weissmann N, Rose F, Akkayagil E, Manz D, Grimminger F, Seeger W, Bohle RM (2002) cDNA array hybridization after laser-assisted microdissection from nonneoplastic tissue. Am J Pathol 160:81-90

22. Silverman J, Muir WW 3rd (1993) A review of laboratory animal anesthesia with chloral hydrate and chloralose. Lab Anim Sci 43:210-216

23. Wilhelm J, Muyal JP, Best J, Kwapiszewska G, Stein MM, Seeger W, Bohle RM, Fink L (2006) Systematic comparison of the T7-IVT and SMART-based RNA preamplification techniques for DNA microarray experiments. Clin Chem 52:1161-1167
24. Schneider J, Buness A, Huber W, Volz J, Kioschis P, Hafner M, Poustka A, Sültmann H (2004) Systematic analysis of T7 RNA polymerase based in vitro linear RNA amplification for use in microarray experiments. BMC Genomics 5:29

25. Puskás LG, Zvara A, Hackler L Jr, Van Hummelen P (2002) RNA amplification results in reproducible microarray data with slight ratio bias. Biotechniques 32:1330-1334, 1336, 1338, 1340

26. Carrol ED, Mankhambo LA, Balmer P, Nkhoma S, Banda DL, Guiver M, Jeffers G, Makwana N, Molyneux EM, Molyneux ME, Smyth RL, Hart CA, Invasive Pneumococcal Disease Study Group (2007) Chemokine responses are increased in HIV-infected Malawian children with invasive pneumococcal disease. J Acquir Immune Defic Syndr 44:443-450

27. Riese J, Schoolmann S, Denzel C, Herrmann O, Hohenberger W, Haupt W (2002) Effect of abdominal infections on peritoneal and systemic production of interleukin 6 and monocyte chemoattractant protein-1. Shock 17:361-364

28. Bossink AW, Paemen L, Jansen PM, Hack CE, Thijs LG, Van Damme J (1995) Plasma levels of the chemokines monocyte chemotactic proteins- 1 and -2 are elevated in human sepsis. Blood 86:3841-3847

29. Kalehua AN, Nagel JE, Whelchel LM, Gides JJ, Pyle RS, Smith RJ, Kusiak JW, Taub DD (2004) Monocyte chemoattractant protein-1 and macrophage inflammatory protein- 2 are involved in both excitotoxin-induced neurodegeneration and regeneration. Exp Cell Res 297:197-211

30. Eugenin EA, D'Aversa TG, Lopez L, Calderon TM, Berman JW (2003) MCP-1 (CCL2) protects human neurons and astrocytes from NMDA or HIV-tatinduced apoptosis. J Neurochem 85:1299-1311

31. Dimitrijevic OB, Stamatovic SM, Keep RF, Andjelkovic AV (2007) Absence of the chemokine receptor CCR2 protects against cerebral ischemia/reperfusion injury in mice. Stroke 38:1345-1353

32. Abraham E, Kaneko DJ, Shenkar R (1999) Effects of endogenous and exogenous catecholamines on LPSinduced neutrophil trafficking and activation. Am J Physiol 276:L1-L8
33. Hofstetter C, Boost KA, Hoegl S, Flondor M, Scheller B, Muhl H, Pfeilschifter J, Zwissler B (2007) Norepinephrine and vasopressin counteract anti-inflammatory effects of isoflurane in endotoxemic rats. Int $\mathbf{J}$ Mol Med 20:597-604

34. Tsuda Y, Kobayashi M, Herndon DN, Suzuki F (2008) Impairment of the host's antibacterial resistance by norepinephrine activated neutrophils. Burns 34:460-466

35. Blandino P Jr, Barnum CJ, Deak T (2006) The involvement of norepinephrine and microglia in hypothalamic and splenic IL-1beta responses to stress. J Neuroimmunol 173:87-95

36. Conte D, Holcik M, Lefebvre CA, Lacasse E, Picketts DJ, Wright KE, Korneluk RG (2006) Inhibitor of apoptosis protein cIAP2 is essential for lipopolysaccharide-induced macrophage survival. Mol Cell Biol 26:699-708

37. Tissier S, Lancel S, Marechal X, Mordon S, Depontieu F, Scherpereel A, Chopin C, Neviere R (2004) Calpain inhibitors improve myocardial dysfunction and inflammation induced by endotoxin in rats. Shock 21:352-357

38. Scherpereel A, Depontieu F, Grigoriu B, Cavestri B, Tsicopoulos A, Gentina $\mathrm{T}$, Jourdain M, Pugin J, Tonnel AB, Lassalle P (2006) Endocan, a new endothelial marker in human sepsis. Crit Care Med 34:532-537

39. Semmler A, Okulla T, Sastre M, Dumitrescu-Ozimek L, Heneka MT (2005) Systemic inflammation induces apoptosis with variable vulnerability of different brain regions. J Chem Neuroanat 30:144-157 